\title{
Prognostic factors associated with locally advanced gastric cancer patients treated with neoadjuvant chemotherapy followed by surgical resection
}

\author{
Yongkun Sun ${ }^{1}$, Lin Yang $^{1}$, Chengfeng Wang ${ }^{1}$, Dongbing Zhao ${ }^{1}$, Jianqiang Cai ${ }^{1}$, \\ Wenbin $\mathrm{Li}^{1}$, Wen Zhang ${ }^{1}$, Jing Huang ${ }^{1}$ and Aiping Zhou ${ }^{1}$ \\ ${ }^{1}$ National Cancer Center/Cancer Hospital, Chinese Academy of Medical Sciences \& Peking Union Medical College, Beijing, China \\ Correspondence to: Lin Yang, email: lyang69@sina.com \\ Keywords: prognostic factors, locally advanced gastric cancer, neoadjuvant chemotherapy, gastrectomy \\ Received: February 07, $2017 \quad$ Accepted: July 25, $2017 \quad$ Published: September 06, 2017 \\ Copyright: Sun et al. This is an open-access article distributed under the terms of the Creative Commons Attribution License 3.0 \\ (CC BY 3.0), which permits unrestricted use, distribution, and reproduction in any medium, provided the original author and source \\ are credited.
}

\section{ABSTRACT}

In this retrospective study, we analyzed prognostic factors associated with survival outcomes in 73 locally advanced gastric cancer patients treated with neoadjuvant chemotherapy (NAC) followed by surgical resection. Median diseasefree survival (DFS) for 64 patients that received $R 0$ resection was 685 days, whereas median overall survival (OS) for 73 patients was 930 days. Multivariate analysis demonstrated that post-treatment nodal stages $(P=0.002)$, nervous invasion $(P=0.0492)$ and serum CA199 levels $(P=0.0398)$ were independent prognostic factors for DFS. Nodal stages $(P=0.0007)$, presence of nervous invasion $(P=\mathbf{0 . 0 2 5 9})$ and non-radical resection $(P=0.0165)$ were independent prognostic factors for $O S$. These results indicate that post-treatment nodal stages, neural invasion and serum CA199 levels are all associated with poor DFS. Moreover, post-treatment nodal stage, resection type and neural invasion status are independent prognostic factors for $0 S$.

\section{INTRODUCTION}

Gastric cancer ranks fifth among most malignant cancers and third among cancer related deaths worldwide [1]. China has the highest incidence of gastric cancer accounting for $35 \%$ of total gastric cancer cases worldwide with a high mortality rate of 25.16 cases per 100,000 [2]. Prognosis of locally advanced gastric cancer is poor with a 5-year overall survival (OS) rate of $20-30 \%$ for surgery-only patients [3]. Neoadjuvant chemotherapy (NAC) is preferred for locally advanced gastric cancer patients since the release of the MRC Adjuvant Gastric Infusional Chemotherapy (MAGIC) trial results [4]. Many studies demonstrate that NAC reduces the size of gastric cancer lesions thereby decreasing tumor staging and increasing the chances for radical resection and survival while decreasing post-operative complications [5-9]. However, standard regimen and courses of NAC are not yet established and prognostic factors associated with survival outcomes for patients treated with NAC followed by surgery remain unclear.
Therefore, we analyzed the relevant prognostic factors associated with survival by reviewing medical records of 73 locally advanced gastric cancer patients that were treated with neoadjuvant chemotherapy followed by curative-intent surgery. Our analysis included the prognostic status of pathological response to NAC and post-therapy node status (Nodal stage or lymph node metastasis ratio).

\section{RESULTS}

Patient and treatment characteristics

Table 1 shows patient and treatment characteristics. The median patient age was 53.0 years (range:32-77 yrs), and 48 of the $73(65.8 \%)$ patients were male. While all73 patients received at least one cycle of NAC, $37(50.7 \%)$ and $36(49.3 \%)$ patients received doublet and triplet NAC regimen, respectively. Among the 73 patients, 50 (68.5\%) and $20(27.4 \%)$ patients showed tumor partial response (PR) and stable disease (SD), respectively. However, 
Table 1: Patient and treatment characteristics

\begin{tabular}{ll}
\hline Characteristics & No. of patients
\end{tabular}

Proportion

Gender

Male

$65.8 \%$

Female

$34.2 \%$

Age at diagnosis

$<60$

$64.4 \%$

$\geq 60$

$35.6 \%$

NAC regimen

Doublet

$50.7 \%$

Triplet

Clinical response to NAC (RECIST)*

SD

$27.4 \%$

PR

Type of resection

R0

64

$87.7 \%$

R1

$6.8 \%$

R2

Graded pathologic response

Minor

$54.8 \%$

Moderate

$26.0 \%$

Major

$19.2 \%$

N stage

N0

$26.0 \%$

N1

$20.5 \%$

N2

$17.8 \%$

N3

Lymph node metastasis ratio

NR0

NR1

NR2

$26.0 \%$

NR3

vascular invasion:

Yes

No

Nervous invasion§

Yes

$11.0 \%$

No

$87.7 \%$ 


\begin{tabular}{lcc}
\hline Characteristics & No. of patients & Proportion \\
\hline Adjuvant Chemotherapy $\|$ & 51 & $70.0 \%$ \\
Yes & 22 & \\
No & & \\
Postoperative Radiotherapyq & 8 & $11.0 \%$ \\
Yes & 65 & \\
No & 65 & \\
\hline
\end{tabular}

* Tumor response to NAC was not evaluated for 3 patients; 2 of them developed ileus during treatment, and the other one missed assessment for personal reasons.

$\$ / \S$ vascular invasion and nervous invasion status were not reported for one patient.

|| 51 patients were administered post-surgery chemotherapy and had medical records in our hospital;The post-surgery chemotherapy records of 22 patients was not known.

I 8 patients received post-operative radiotherapy.

tumor response was not evaluated for $3(4.1 \%)$ patients because 2 patients developed ileus and one patient missed assessment due to personal reasons. Of the 73 patients that had underwent NAC, $64(87.7 \%)$ underwent complete resection with negative margins (R0), $5(6.8 \%)$ underwent microscopic resection (R1) and 4 (5.5\%) patients underwent resection with grossly positive margins (R2). While $51(70.0 \%)$ patients received post-operative chemotherapy in our hospital, there was no record for 22 $(30 \%)$ patients. Adjuvant chemotherapy (AC) regimen in 23 patients was the same as NAC. Eight(11\%) patients received post-operative radiotherapy. Median follow-up period was 635 days (range 123 - 1962 days) with Aug 20,2013as the cutoff date. At this time, 37 patients were still alive with 29 out of the 37 showing no documented progression.

\section{Prognostic factors associated with disease-free survival}

Median DFS for 64 patients that received R0 resection was 685 days (Figure 1). Univariate analysis showed that nodal stages, lymph node metastasis ratio and nervous invasion were prognostic factors associated with DFS (Table 2). Patients with N0, N1 and N3 stages showed decreasing DFS rates (923, 630, and 263 days, respectively; $\mathrm{P}<0.0001$; insufficient data to estimate DFS in N2 stage patients). Patients with lymph node metastasis

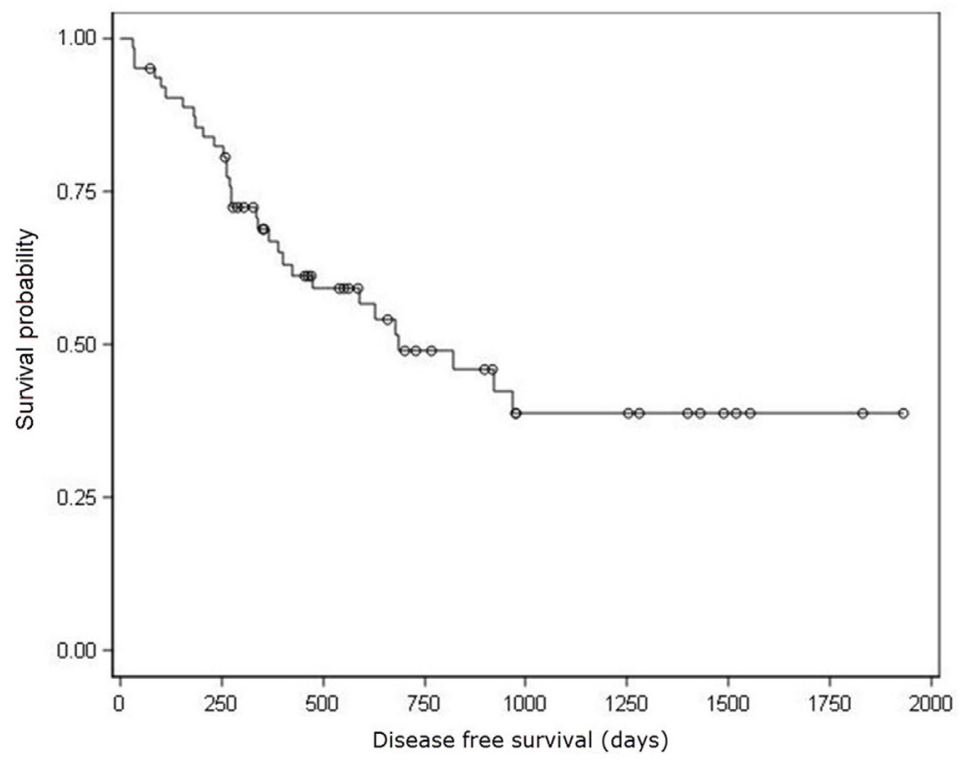

Figure 1: Kaplan-Meier analysis of disease free survival. $\circ$ Censored Patients. Median DFS for 64 patients that received R0 resection was 685 days. 
Table 2: Univariate prognostic factors analyses of disease-free and overall survival

\begin{tabular}{|c|c|c|c|c|c|c|}
\hline \multirow{2}{*}{ Variable } & \multicolumn{2}{|c|}{ DFS } & \multirow{2}{*}{ P value } & \multicolumn{2}{|l|}{ OS } & \multirow{2}{*}{ P value } \\
\hline & No. of patients & Median & & No. of patients & Median & \\
\hline \multicolumn{7}{|c|}{ Age at diagnosis } \\
\hline$\geq 60$ & 23 & 679 & 0.8901 & 26 & 878 & 0.4526 \\
\hline$<60$ & 41 & 658 & & 47 & 953 & \\
\hline \multicolumn{7}{|l|}{ Gender } \\
\hline Male & 43 & 685 & 0.5737 & 48 & 878 & 0.9442 \\
\hline Female & 21 & 630 & & 25 & 930 & \\
\hline \multicolumn{7}{|l|}{ CEA } \\
\hline Abnormal & 13 & 426 & 0.5313 & 18 & 564 & 0.0400 \\
\hline Normal & 46 & 821 & & 50 & 1039 & \\
\hline \multicolumn{7}{|l|}{ CA199 } \\
\hline Abnormal & 15 & 402 & 0.0613 & 19 & 624 & 0.0254 \\
\hline Normal & 44 & 923 & & 49 & 1165 & \\
\hline \multicolumn{7}{|l|}{ CA724 } \\
\hline Abnormal & 14 & 304 & 0.1402 & 17 & 624 & 0.2505 \\
\hline Normal & 40 & 630 & & 46 & 878 & \\
\hline \multicolumn{7}{|c|}{$\begin{array}{l}\text { Neoadjuvant chemotherapy } \\
\text { regimen }\end{array}$} \\
\hline Doublet & 30 & 821 & 0.7547 & 37 & 878 & 0.3531 \\
\hline Triplet & 34 & 679 & & 36 & 1039 & \\
\hline \multicolumn{7}{|l|}{ RECIST } \\
\hline SD & 15 & 923 & 0.3229 & 20 & 806 & 0.3351 \\
\hline PR & 47 & 630 & & 50 & 930 & \\
\hline \multicolumn{7}{|c|}{ Graded pathologic response } \\
\hline Minor & 31 & 821 & 0.9908 & 40 & 752 & 0.0758 \\
\hline Moderate & 19 & 591 & & 19 & 1039 & \\
\hline Major & 14 & 685 & & 14 & $\mathrm{NE} \dagger$ & \\
\hline \multicolumn{7}{|c|}{ Resection Type } \\
\hline R0 & 64 & 685 & $\mathrm{NA}^{*}$ & 64 & 1165 & $<0.0001$ \\
\hline $\mathrm{R} 1+\mathrm{R} 2$ & NA & NA* & & 9 & 452 & \\
\hline \multicolumn{7}{|c|}{ Nodal stage } \\
\hline N3 & 19 & 263 & $<0.0001$ & 26 & 550 & $<0.0001$ \\
\hline $\mathrm{N} 2$ & 12 & $\mathrm{NE} \dagger$ & & 13 & $\mathrm{NE} \dagger$ & \\
\hline N1 & 15 & 630 & & 15 & 1165 & \\
\hline N0 & 18 & 923 & & 19 & $\mathrm{NE} \dagger$ & \\
\hline \multicolumn{7}{|c|}{ Lymph node metastasis ratio } \\
\hline NR3 & 12 & 268 & 0.0022 & 12 & 604 & 0.0215 \\
\hline NR2 & 19 & 679 & & 19 & 1624 & \\
\hline
\end{tabular}




\begin{tabular}{|c|c|c|c|c|c|c|}
\hline \multirow{2}{*}{ Variable } & \multicolumn{2}{|c|}{ DFS } & \multirow{2}{*}{ Pvalue } & \multicolumn{2}{|l|}{ OS } & \multirow{2}{*}{ P value } \\
\hline & No. of patients & Median & & No. of patients & Median & \\
\hline NR1 & 15 & 821 & & 15 & 1165 & \\
\hline NR0 & 18 & 923 & & 27 & 953 & \\
\hline \multicolumn{7}{|c|}{ Vascular invasion } \\
\hline Yes & 15 & 388 & 0.0666 & 19 & 604 & 0.0782 \\
\hline No & 48 & 923 & & 53 & 1431 & \\
\hline \multicolumn{7}{|c|}{ Nervous invasion } \\
\hline Yes & 5 & 185 & 0.0139 & 8 & 443.5 & 0.0003 \\
\hline No & 58 & 821 & & 64 & 1039 & \\
\hline \multicolumn{7}{|c|}{$\begin{array}{l}\text { if } \mathrm{AC} \text { and } \mathrm{NAC} \\
\text { regimensaresimilar }\end{array}$} \\
\hline Yes & 21 & 679 & 0.7393 & 23 & 930 & 0.7334 \\
\hline No & 43 & 821 & & 50 & 878 & \\
\hline
\end{tabular}

*NAs are not applicable; $\uparrow$ NEs not estimated because of insufficient survival data.

ratiosNR0, NR1, NR2, and NR3also demonstrated decreasing DFS rates (923, 821, 679 and 267 days, respectively; $\mathrm{P}=0.0022$ ). Patients with nervous invasion showed lower DFS (185 days) than patients without nervous invasion (821 days; $\mathrm{P}=0.0139$ ). Furthermore, vascular invasion and elevated serum CA199 levels were marginally associated with shorter DFS $(\mathrm{P}=0.0666$ and $\mathrm{P}$ $=0.0613$, respectively) .

Multivariate analysis by stepwise Cox model showed that post-treatment nodal stage, nervous invasion and serum CA199 levels were independent prognostic factors for DFS (Table 3). Higher N stages [N1 (HR $=2.028,95 \% \mathrm{CI}=0.604-6.808), \mathrm{N} 2(\mathrm{HR}=0.812,95 \% \mathrm{CI}$ $=0.187-3.530)$, and N3 ( $\mathrm{HR}=7.044,95 \% \mathrm{CI}=2.189$ 22.666), $\mathrm{P}=0.002]$, nervous invasion $(\mathrm{HR}=3.647 ; 95 \%$ $\mathrm{CI}=1.004-13.242 ; \mathrm{P}=0.0492)$, elevated serumCA199(HR $=2.540 ; 95 \% \mathrm{CI}=1.044-6.176 ; \mathrm{P}=0.0398$ ) levels showed significant association with shorter DFS. In contrast, lymph node metastasis ratio and presence of vascular invasion were not associated with DFS according to multivariate analysis.

\section{Prognostic factors associated with overall survival}

Kaplan-Meier survival analysis showed a median OS of 930 days (Figure 2). Univariate analysis for OS showed that nodal stage $(\mathrm{N})$, lymph node metastasis ratio (NR), nervous invasion, resection type and serum CEA/ CA199ratiowere prognostic indicators of OS, whereas, graded pathologic response and presence of vascular invasion showed marginal association with OS (Table 2).
Multivariate analyses showed that higher nodal stages [N1 $(\mathrm{HR}=7.869,95 \% \mathrm{CI}=2.191-28.266)$, N2 (HR $=0.761,95 \% \mathrm{CI}=0.144-4.024)$ and N3 $(\mathrm{HR}=2.923$, $95 \% \mathrm{CI}=0.648-13.185) ; \mathrm{P}=0.0007]$, presence of nervous invasion $(\mathrm{HR}=3.283 ; 95 \% \mathrm{CI}=1.154-9.339 ; \mathrm{P}=0.0259)$ and non-radical resection $(\mathrm{HR}=2.807 ; 95 \% \mathrm{CI}=1.207$ $6.526 ; \mathrm{P}=0.0165)$ were independent prognostic factors for poor OS (Table 4).

\section{DISCUSSION}

Patients with potentially resectable gastric cancer are treated with neoadjuvant chemotherapy to improve survival. However, a standard regimen is not clear. Several investigations have shown that patients that received NAC followed by surgery, age at diagnosis, post-treatment nodal status, diffuse-type histology, perineural invasion/vascular invasion and salvage surgery are associated with OS [10 13]. Our study demonstrated that clinical response (SD or PR) was not a significant prognosis factor for DFS or OS. One possible reason is that we enrolled only limited number of patients that underwent curative-intent surgery after NAC.

The prognostic value of pathologic response to neoadjuvant chemotherapy remains controversial although it has been investigated for various malignancies. Kurokawa Y et al. suggested that pathological response was a better surrogate endpoint than RECIST in neoadjuvant chemotherapy for gastric cancer [14]. In many studies, univariate analysis showed that pathologic response was a predictor of survival in GC patients after receiving neoadjuvant chemotherapy; but, multivariate 
Table 3: Multivariate analysis of prognostic factors for disease-free survival by stepwise Cox model

\begin{tabular}{lccc}
\hline Variable & Hazard Ratio & 95\% CI & P value \\
\hline Nervous invasion & 3.647 & $(1.004-13.242)$ & 0.0492 \\
Yes & 1 & & 0.0020 \\
No & & $(2.189-22.666)$ & $(0.187-3.530)$ \\
Nodal stage & 7.044 & $(0.604-6.808)$ & - \\
N3 & 0.812 & $(1.044-6.176)$ & 0.0398 \\
N2 & 2.028 & - \\
N1 & 1 & & \\
N0 & & & \\
Abnormal & 2.540 & 1 & \\
Normal & & & \\
\hline
\end{tabular}

analysis showed that it was not an independent predictor of OS [10, 15-18]. In this study, univariate analysis showed that pathologic response was marginally associated with OS, but not associated with DFS; multivariate analysis showed that it was not associated with both DFS and OS. Fujitaniet al. showed that pathologic response was associated with OS in the subset of patients with nodal stages N0-1 [10]. Therefore, further prospective studies with larger sample size are necessary to confirm the prognostic status of pathologic response in patients that undergo curative-intent surgery after NAC.

Metastatic lymph node ratio (NR) is an alternate prognostic factor instead of the number of lymph nodes ( $\mathrm{N}$ in TNM staging) in GC because of the limited number of lymph nodes $[18,19]$. Persiani $\mathrm{R}$ et al. showed that TRM ( $\mathrm{R}$ means metastatic lymph node ratio) staging system had better prognostic power than the TNM system by reviewing 219 patients that underwent gastrectomy

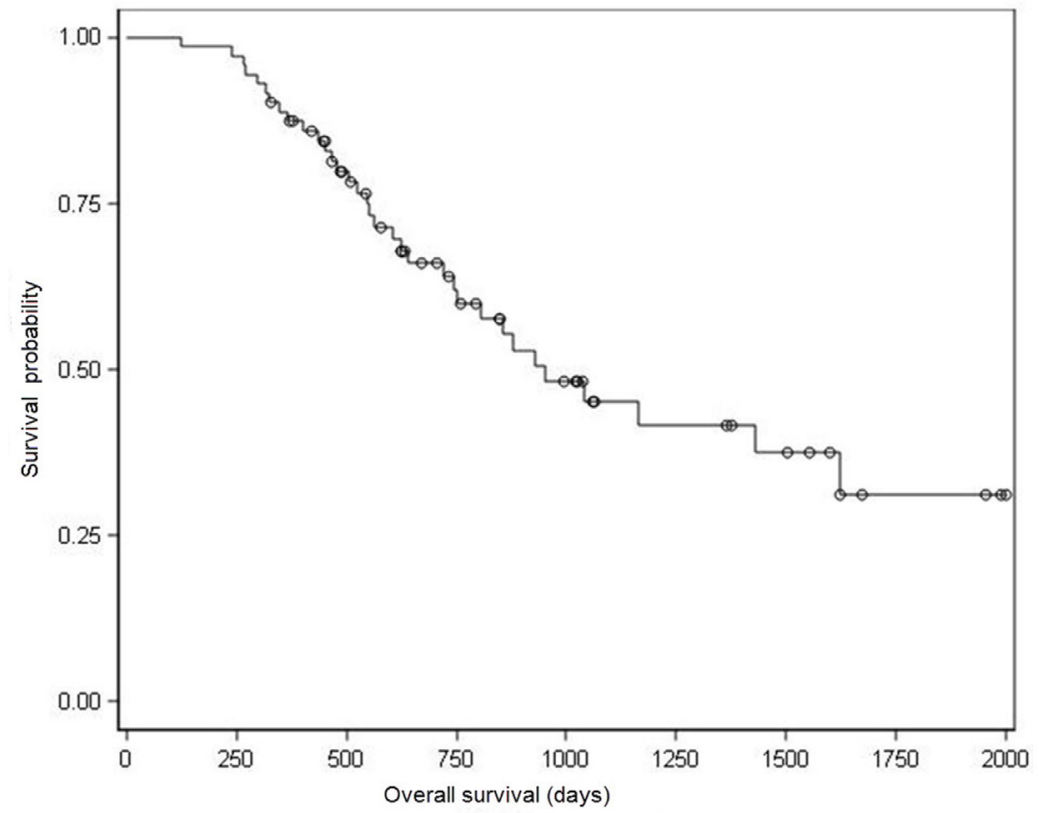

Figure 2: Kaplan-Meier analysis of overall survival. $\circ$ Censored Patients. Median OS for all of these 73 patients was 930 days. 
Table 4: Multivariate analysis of prognostic factors for overall survival by the stepwise Cox's model

\begin{tabular}{lccc}
\hline Variable & Hazard Ratio & 95\% CI & P value \\
\hline Nodal stage & 7.869 & $(2.191,28.266)$ & 0.0007 \\
N3 & 0.761 & $(0.144,4.024)$ & $(0.648,13.185)$ \\
N2 & 2.923 & - \\
N1 & 1 & $(1.154,9.339)$ & - \\
N0 & & -0259 \\
Nervous invasion & 3.283 & $(1.207,6.526)$ \\
Yes & 1 & - \\
No & & & 0.0165 \\
Resection type & 2.807 & & \\
R1+R2 & 1 & & \\
R0 & &
\end{tabular}

for node-positive (M0) cancer [20]. Posteraro B et al. retrospectively reviewed 110 patients that received curative-intent gastrectomy by the TRM staging and demonstrated that higher NR strongly predicted poor OS and DFS [21]. In the present study, NR was a prognostic factor for both DFS and OS in univariate analysis, but was in significant in multivariable analysis. However, posttreatment $\mathrm{N}$ stage showed association with DFS and OS in both univariable and multivariable analyses, consistent with the study by Fujitani et al.[10].Therefore, posttreatment $\mathrm{N}$ stage was a more reliable prognostic factor than metastatic lymph node ratio (NR) in locally advanced GC patients that received gastrectomy after NAC.

Changes in serum CA199 levels demonstrate therapeutic efficacy with increased serum CA199 levels indicating treatment failure or recurrence. Mohri et al. showed thatCA199 was an independent prognostic factor for OS in patients with metastatic gastric cancer [22]. Schauer et al demonstrated that serum CA199 levels predicted survival in patients with diffuse type gastric cancer after surgical treatment [23]. Zhu et al showed that serum CA199 was part of the prognostic index for patients with metastatic gastric cancer that received epirubicin (EPR)-containing triplet regimen as first-line treatment [24]. In our study, univariate analysis showed that elevated serumCA199 levels were associated with both shorter DFS and OS, but multivariate analysis showed that it was associated with poor DFS. This suggested that serum CA199 levels were strong predictors of long-term survival inGC patients.

Our findings are limited because this was a retrospective study conducted in a single institution with few select patients (73 study subjects) with different preand post-operative chemotherapeutic regimens. Larger multi-center prospective studies are necessary to confirm our findings.

In conclusion, our study demonstrates that posttreatment nodal stages, neural invasion and serum CA199 levels are associated with poor DFS. Moreover, posttreatment nodal stage, resection type and neural invasion status are independent prognostic factors for OS. This study also revealed that post-treatment $\mathrm{N}$ stage was a more reliable prognostic factor than metastatic lymph node ratio (NR) in locally advanced GC patients that received gastrectomy after NAC.

\section{MATERIALS AND METHODS}

\section{Patients and treatment schedule}

We retrospectively analyzed the outcomes of 73 consecutive patients with locally advanced gastric cancer that were treated with neoadjuvant chemotherapy followed by surgical resection between August 2007 and July 2012 at the National Cancer Center/Cancer Hospital, Chinese Academy of Medical Sciences \& Peking Union Medical College, China. The procedures followed were in accordance with the ethical standards set by the independent ethnic committee of Cancer Hospital of Chinese Academy of Medical Sciences \& Peking Union Medical College on human experimentation and the Helsinki Declaration. The patients were diagnosed with resectable advanced adenocarcinoma (gastric cancer) and completed at least one course of NAC with the tumor response evaluated by Response Evaluation Criteria in Solid Tumors (RECIST, version 1.0). All patients received gastrectomy, with D2 lymph-node dissection. The posttherapy node status including number of metastatic lymph nodes ( $\mathrm{N}$ stage) and lymph node metastasis ratio (NR) 
were determined after examination. The extent of residual tumor was determined by the pathologist in our hospital to estimate the pathologic response to NAC. After surgery, patients received post-operative chemotherapy and/or radiotherapy based on the clinical status of individual patients. The patients followed up by regular clinic visits and phone calls.

\section{Statistical analysis}

SAS statistical software 9.3 (SAS Institute Inc., Cary, NC, USA) was used for all statistical analyses. Overall survival (OS) was defined as the time from the date of initiation of NAC until death from any cause (event) or the last follow-up date (censored). Disease-free survival (DFS) was defined as the time from the date of surgery until local or distant relapse was detected (event) or the last follow-up date (censored). Kaplan Meier survival analysis was used to determine both DFS and OS.

Univariate analyses were performed by the log rank test for the following variables: age at diagnosis ( $\geq 60$ vs. $\leq 60$ ); gender (male vs. female); abnormal versus normal levels of serum CEA, CA199and CA724; Neoadjuvant chemotherapy regimen [doublet (Fluoropyrimidine + Platinum) versus triplet (Fluoropyrimidine + Platinum + Anthracyclines or Taxane)]; clinical response (partial response vs. stable disease); graded pathologic response (minor vs. moderate vs. major); resection type (R0 vs. R1+R2); Nodal stage [N0 (no metastasis), N1 (1-2 metastatic nodes), N2 (3-6 metastatic nodes), or N3 (7 or more metastatic nodes)]; lymph node metastasis ratio [number of positive nodes/number of nodes examined and classified as NR0 (0\%), NR1 (<15\%), NR2 (15-40\%), or NR3 (>40\%)];presence or absence of vascular invasion; presence or absence of nervous invasion; and if regimen of AC was the same as NAC or not.

Multivariate analysis of prognostic factors was performed by the stepwise Cox proportional hazard model with the variables identified as significant factors in the univariate analyses and hazard ratio (HR) and their95\% confidence intervals (CIs) were calculated. A two-sided P $<0.05$ was considered statistically significant.

\section{Author contribution}

All authors contributed equally to this work and agreed with the contents of the manuscript.

\section{CONFLICTS OF INTEREST}

The authors declare that they have no conflicts of interest.

\section{REFERENCES}

1. GLOBOCAN 2012. Estimated cancer incidence, mortality and prevalence worldwide in 2012

2. Zhou XN, Sun XB. Analysis of the incidence and mortality of gastric cancer in China during 2003-2007. Cancer. 2012; 32:109-114.

3. Ronellenfitsch U, Schwarzbach M, Hofheinz R, Kienle P, Kieser M, Slanger TE, Burmeister B, Kelsen D, Niedzwiecki D, Schuhmacher C, Urba S, van de Velde C, Walsh TN, et al. Preoperative chemo (radio) therapy versus primary surgery for gastroesophageal adenocarcinoma: systematic review with meta-analysis combining individual patient and aggregate data. Eur J Cancer. 2013; 49:3149-58.

4. Cunningham D, Allum WH, Stenning SP, Thompson JN, Van de Velde CJ, Nicolson M, Scarffe JH, Lofts FJ, Falk SJ, Iveson TJ, Smith DB, Langley RE, Verma M, et al. Perioperative chemotherapy versus surgery alone for resectable gastroesophagealcancer. N Engl J Med. 2006; 355:11-20.

5. Xiong B, Ma L, Cheng Y, Zhang C. Clinical effectiveness of neoadjuvant chemotherapy in advanced gastric cancer: an updated meta-analysis of randomized controlled trials. Eur J Surg Oncol. 2014; 40:1321-30.

6. Hashemzadeh S, Pourzand A, Somi MH, Zarrintan S, Javad-Rashid R, Esfahani A. The effects of neoadjuvant chemotherapy on resectability of locally-advanced gastric adenocarcinoma: a clinical trial. Int J Surg. 2014; 12:1061-9.

7. Boige V, Pignon J, Saint-Aubert B, Lasser P, Conroy T, Bouché O, Segol P, Bedenne L, Rougier P, Ychou M. Final results of a randomized trial comparing preoperative 5-fluorouracil (F)/cisplatin (P) to surgery alone in adenocarcinoma of stomach and lower esophagus (ASLE): FNLCC ACCORD07-FFCD 9703 trial. J Clin Oncol. 2007; 25(18 suppl):4510.

8. Schuhmacher C, Gretschel S, Lordick F, Reichardt P, Hohenberger W, Eisenberger CF, Haag C, Mauer ME, Hasan B, Welch J, Ott K, Hoelscher A, Schneider PM, et al. Neoadjuvant chemotherapy compared with surgery alone for locally advanced cancer of the stomach and cardia: European Organisation for Research and Treatment of Cancer randomized trial 40954. J Clin Oncol. 2010; 28:5210-8.

9. Tian SB, Yu JC, Kang WM, Ma ZQ, Ye X, Yan C, Huang YK. Effect of Neoadjuvant Chemotherapy Treatment on Prognosis of Patients with Advanced Gastric Cancer: A Retrospective Study. Chin Med Sci J. 2015; 30:84-9.

10. Fujitani K, Mano M, Hirao M, Kodama Y, Tsujinaka T. Posttherapy nodal status, not graded histologic response, predicts survival after neoadjuvant chemotherapy for advanced gastric cancer. Ann Surg Oncol. 2012; 19:1936-43. 
11. Jhawer M, Coit D, Brennan M, Qin LX, Gonen M, Klimstra D, Tang L, Kelsen DP, Shah MA. Perineural invasion after preoperative chemotherapy predicts poor survival in patients with locally advanced gastric cancer: gene expression analysis with pathologic validation. Am J Clin Oncol. 2009; 32:356-62.

12. Koh YW, Park YS, Ryu MH, Ryoo BY, Park HJ, Yook JH, Kim BS, Kang YK. Postoperative nodal status and diffuse-type histology are independent prognostic factors in resectable advanced gastric carcinomas after preoperative chemotherapy. Am J Surg Pathol. 2013; 37:1022-9.

13. Yano M, Shiozaki H, Inoue M, Tamura S, Doki Y, Yasuda T, Fujiwara Y, Tsujinaka T, Monden M. Neoadjuvant chemotherapy followed by salvage surgery: effect on survival of patients with primary noncurative gastric cancer. World J Surg. 2002; 26:1155-9.

14. Kurokawa Y, Shibata T, Sasako M, Sano T, Tsuburaya A, Iwasaki Y, Fukuda H. Validity of response assessment criteria in neoadjuvant chemotherapy for gastric cancer (JCOG0507-A). Gastric Cancer. 2014; 17:514-21.

15. Becker K, Mueller JD, Schulmacher C, Ott K, Fink $\mathrm{U}$, Busch R, Böttcher K, Siewert JR, Höfler $H$. Histomorphology and grading of regression in gastric carcinoma treated with neoadjuvant chemotherapy. Cancer. 2003; 98:1521-30.

16. Mansour JC, Tang L, Shah M, Bentrem D, Klimstra DS, Gonen M, Kelsen DP, Brennan MF, Coit DG. Does graded histologic response after neoadjuvant chemotherapy predict survival for completely resected gastric cancer? Ann Surg Oncol. 2007; 14:3412-8.

17. Ajani JA, Winter K, Okawara GS, Donohue JH, Pisters PW, Crane CH, Greskovich JF, Anne PR, Bradley JD, Willett C, Rich TA. Phase II trial of preoperative chemoradiation in patients with localized gastric adenocarcinoma (RTOG
9904): quality of combined modality therapy and pathologic response. J Clin Oncol. 2006; 24:3953-8.

18. Lee SY, Hwang I, Park YS, Gardner J, Ro JY. Metastatic lymph node ratio in advanced gastric carcinoma: a better prognostic factor than number of metastatic lymph nodes? Int J Oncol. 2010; 36:1461-7.

19. Persiani R, Rausei S, Biondi A, Boccia S, Cananzi F, D’Ugo D. Ratio of metastatic lymph nodes: impact on staging and survival of gastric cancer. Eur J Surg Oncol. 2008; 34:519-24.

20. Persiani R, Rausei S, Antonacci V, Biondi A, Casella F, Ciccoritti L, D’Ugo D. Metastatic Lymph Node Ratio: A New Staging System for Gastric Cancer. World J Surg. 2009; 33:2106-2111.

21. Posteraro B, Persiani R, Dall'Armi V, Biondi A, Arzani D, Sicoli F, Bonassi S, D’Ugo D, Ricciardi W, Boccia S. Prognostic factors and outcomes in Italian patients undergoing curative gastric cancer surgery. Eur J Surg Oncol. 2014; 40:345-51.

22. Mohri Y, Tanaka K, Ohi M, Saigusa S, Yasuda H, Toiyama Y, Araki T, Inoue Y, Kusunoki M. Identification of prognostic factors and surgical indications for metastatic gastric cancer. BMC Cancer. 2014; 14:409.

23. Schauer M, Peiper M, Theisen J, Knoefel W. Prognostic factors in patients with diffuse type gastric cancer (linitis plastica) after operative treatment. Eur J Med Res. 2011; 16:29-33.

24. Zhu X, Zhao X, Peng W, Sun S, Cao J, Ji D, Liu X, Wang C, Huang M, Yu H, Guo W, Li J, Yin J. Epirubicin combined with oxaliplatin and 5-day continuous infusion of 5-fluorouracil as a first-line treatment for metastatic gastric cancer: treatment outcomes and analysis of prognostic factors. J Cancer Res Clin Oncol. 2015; 141:109-18. 